\title{
DESIGUALDADES ENTRE MUJERES Y HOMBRES EN TODOS LOS CICLOS DE VIDA. EVIDENCIAS DE EXCLUSIÓN SOCIAL EN LA REPÚBLICA DOMINICANA: UNA NOTA CRÍTICA
}

\section{Inequalities between women and men in all life cycles Evidences of social exclusion in the Dominican Republic: a critical note}

\section{Centro de Estudios de Género}

Instituto Tecnológico de Santo Domingo (INTEC). Área de Ciencias Sociales y Humanidades. Correo de correspondencia: Lourdes Contreras, directora del Centro de Estudios de Género (INTEC).

lourdes.contreras@intec.edu.do

Recibido: $10 / 4 / 2018 \bullet$ Aprobado: 14/5/2018

Cómo citar: Centro de Estudios de Género del INTEC. (2018). Desigualdades entre mujeres y hombres en todos los ciclos de vida. Evidencias de exclusión social en la República Dominicana: una nota crítica. Ciencia y Sociedad, 43(2), 67-80.

doi: http://dx.doi.org/10.22206/cys.2018.v43i2.pp67-80

\section{Resumen}

El 8 de marzo de cada año se conmemora, de manera especial, la lucha de las mujeres del mundo en contra de la desigualdad. En este año 2018, en la República Dominicana continuamos viviendo desigualdades e injusticias históricas que afectan a la mayoría de la población y que son vividas en mayor profundidad por las mujeres, independientemente del ámbito territorial, urbano o rural y del ciclo de vida en que se encuentren -niñas, jóvenes, adultas o adultas mayores-.

En el análisis que presenta este documento del CEGINTEC se presenta información clave sobre las tendencias que se observan en la situación por la que atraviesan mujeres y niñas de zonas urbanas y rurales en República Dominicana en el presente año 2018, en comparación con los hombres, y cómo esos datos son el reflejo de grandes retrasos para el ejercicio de los derechos de las mujeres y para el desarrollo humano del país.

Palabras claves: desigualdad social; derechos de la mujer; desarrollo humano; violencia; pobreza.

\begin{abstract}
On March 8 of each year, the struggle of the women of the world against inequality is commemorated in a special way. In this year 2018, in the Dominican Republic, we continue to experience inequalities and historical injustices that affect the majority of the population and that are lived in greater depth by women, regardless of the territorial, urban or rural scope and the life cycle in which they live. find -children, young people, adults or older adults-.

The analysis presented in this document of the CEGINTEC presents key information on the trends observed in the situation of women and girls in urban and rural areas in the Dominican Republic in the current year 2018, compared to men, and how these data reflect long delays in the exercise of women's rights and human development in the country.
\end{abstract}

Keywords: Social Inequality; Women's Rights; Human Development; Violence; Poverty. 
Datos significativos sobre las desigualdades de género en República Dominicana

\section{Distribución desigual del crecimiento económi- co: pobreza y desarrollo humano}

La principal característica del análisis del crecimiento económico en el país es su inequitativa distribución. A pesar de los importantes avances en desarrollo humano en los últimos 25 años, este progreso es lento y desigual en el caso de determinados grupos, especialmente de las mujeres. El país continúa clasificado en el renglón de Desarrollo humano alto, ubicándose en la posición 99 de 188 países, mejorando 2 posiciones con relación al informe de 2015, cuando obtuvo la posición 101. El mejor desempeño se obtuvo en la dimensión de ingresos, seguida por la esperanza de vida al nacer y el acceso a la educación. Sin embargo, el Índice de Desarrollo Humano (IDH) disminuye en un $21.7 \%$, cuando se ajusta por desigualdad, presentando mayor desigualdad en la dimensión de ingreso, seguida por la dimensión de educación y la de salud; reflejando que, a pesar del elevado dinamismo en la generación de riquezas, existen segmentos importantes de la población dominicana que enfrentan limitaciones para beneficiarse de los frutos de tal crecimiento (PNUD, 2016).

Los datos nacionales revelan profundas brechas para el logro del desarrollo humano, especialmente en las personas jóvenes y, sobre todo, en las mujeres. Cuando el nivel de desarrollo humano se examina considerando las desigualdades de género, su valor se reduce en un $47 \%$. De manera particular, se evidencian brechas importantes en la distribución del ingreso, una menor participación de las mujeres en el mercado laboral, la previsión de servicios en la salud sexual y reproductiva, altas tasas de embarazo adolescente (20.3\%), altos índices de mortalidad materna (100 por cada 100 mil nacidos vivos) y baja participación en los asientos congresuales. Otras limitaciones identificadas en el caso de mujeres jóvenes son la oferta de servicios educativos y de salud integral, un mercado laboral excluyente y de baja calidad, bajo acceso a las TIC y acceso restringido a información y servicios de salud sexual y reproductiva (PNUD, 2017).

Los datos del Ministerio de Economía, Planificación y Desarrollo (MEPyD) registran disminuciones en la pobreza moderada y extrema, especialmente desde el año 2013, cuando la pobreza moderada pasó de un $41.3 \%$ a un $30.5 \%$, en 2016 , y la pobreza extrema de un $10.5 \%$ a un $6.5 \%$, en igual período. No obstante, dentro del grupo de personas viviendo en la pobreza, las mujeres son el segmento más vulnerable, al estimarse en un $6.35 \%$ la proporción de mujeres que viven en pobreza extrema a nivel nacional, con mayor afectación a nivel rural (9.49\%), frente al $5.58 \%$ correspondiente a los hombres; mientras que la pobreza moderada afecta al $30.37 \%$ de las mujeres a nivel nacional y al $40.41 \%$ de las que viven en zona rural (MEPyD, 2018) ${ }^{1}$.

Según cifras de CEPAL (2014), los hogares encabezados por mujeres presentan mayor nivel de vulnerabilidad ( $46.5 \%$ en población pobre y $25.0 \%$ en indigentes), mientras que en los hogares con jefatura masculina estos porcentajes son de un 32.8\% en pobreza y un $14.6 \%$ en indigencia. Según el ciclo de vida, los niveles de indigencia y pobreza se concentran en edades entre 15 y 24 años, $(21.6 \%$ en pobreza y $19 \%$ en indigencia); en cambio, en mayores de 65 años los índices son de $42.6 \%$ en pobreza y $22.1 \%$ en indigencia. ${ }^{2}$

1. Ministerio de Economía, Planificación y Desarrollo. (2018). 5to Informe anual de avance en la implementación de la Estrategia nacional de desarrollo 2030 y cumplimiento de los objetivos y metas del Plan plurianual del sector público. Santo Domingo, República Dominicana.

2. Datos al año 2014 reportados por la CEPAL en su página web. Consultada en febrero de 2018. Recuperado de https:// www.cepal.org/es/datos-y-estadisticas. 


\section{Brechas en la autonomía económica}

\section{Trabajo remunerado, empleo y desempleo}

A pesar de las normativas vigentes y de los avances en los niveles educativos de las mujeres, existen importantes brechas en la inserción y las condiciones laborales de estas, con relación a los hombres, lo cual limita el ejercicio de sus derechos económicos y sociales, independientemente de su edad, su nivel educativo, su residencia en zona urbana o rural y su nivel de ingresos.

Las últimas cifras disponibles indican que la población femenina mayor de 15 años sin ingresos propios alcanza el $26.7 \%$ en las zonas urbanas y el $36.4 \%$ en las zonas rurales, mientras que los hombres en igual condición representan el $12.2 \%$ en zonas urbanas y el $13.4 \%$ en zonas rurales ${ }^{3}$. Esto significa que más de la cuarta parte de las mujeres depende de otras personas para su subsistencia, aumentando sus niveles de vulnerabilidad, los cuales se incrementan en las mujeres jóvenes de 15-24 años, cuyo porcentaje llega a un 49\%. En otras palabras, ser mujer y ser mujer joven (15-24 años) significa estar en mayor vulnerabilidad en la República Dominicana.

La tasa de participación de las mujeres en el mercado laboral, si bien ha aumentado en las últimas décadas, todavía registra desigualdades importantes en comparación con los hombres, a pesar de que se produce una ligera tendencia a la baja. Al 2017, solo el $49.1 \%$ de las mujeres mayores de 15 ańos forman parte de la fuerza laboral, mientras que en los hombres esta proporción se sitúa en el $75.9 \%$ (Banco Central de la República Dominicana, $2017)^{4}$. El incremento de la fuerza laboral femenina es atribuida a factores sociodemográficos,

\section{Ibídem}

4. Banco Central de la República Dominicana. Datos publicados en su página web. Consultada en febrero de 2018. Recuperado de https://www.bancentral.gov.do/estadisticas_economicas/mercado_trabajo/ económicos y culturales, como son: el aumento del nivel educativo ${ }^{5}$, la reducción de la tasa de natali$\mathrm{dad}^{6}$, el aumento de la jefatura femenina ${ }^{7}$, la persistencia de altos niveles de vulnerabilidad y pobreza, una mayor conciencia del rol de las mujeres en la generación de ingresos propios, la permanencia de bajos salarios y el deterioro de los salarios reales, lo cual ha requerido que más de un integrante de la familia genere ingresos para solventar las necesidades de la unidad familiar.

Las mujeres entre 20 y 39 años y entre 40 y 59 años tienen las mayores tasas de participación laboral, con el $69.2 \%$ y el $61.9 \%$, respectivamente. De estos porcentajes, el $74.7 \%$ tiene nivel universitario y el $52.4 \%$ nivel de estudios secundarios, quienes a su vez reciben ingresos de $52.5 \%$ y $59.4 \%$.

Los grupos que tienen mayores dificultades para su inserción en el mercado laboral son: las mujeres hasta los 19 años y con más de 60 años; las que tienen menores niveles educativos; las procedentes de hogares de menores ingresos (tasa de actividad de $35.5 \%$ y $41.4 \%$ de los quintiles 1 y 2 ) y las que habitan en zonas rurales (39.6\%) (Ministerio de Economía, Planificación y Desarrollo, 2018).

A pesar de la creciente incorporación de las mujeres al mercado laboral, todavía el 50.9\% de la población femenina mayor de 15 años se reporta como inactiva al 2017. En cambio, en los hombres este porcentaje es de apenas un $24 \%$. La dedicación a

5. La escolaridad promedio de la población de 15 años o más aumentó de 7.3 años en 2000 a 8.8 años en 2015 , donde la educación promedio de las mujeres supera a los hombres, según datos de la ONE. La CEPAL reporta al 2014 una escolaridad promedio para la población masculina de 8.4 años y para la femenina de 10.2 años.

6. La tasa global de natalidad pasó de 3 hijos/as por mujer en 2002 a 2.5 en 2013, según datos reportados por la ONE en su página web https://www.one.gob.do/Categoria/Estadisticas/

7. Se estima una tasa de jefatura femenina de casi un $41.8 \%$ a nivel urbano y un $34.7 \%$ a nivel rural, acorde a la ENDESA, 2013. 
los estudios y al trabajo de los cuidados en el hogar constituyen las principales causas de la ausencia de las mujeres dentro de la población económicamente activa. Esta alta proporción de mujeres en labores del cuidado del hogar es un importante desafío para el Estado dominicano para saldar deudas sociales con las mujeres, considerando aspectos claves como son: el desaprovechamiento de una alta proporción de fuerza laboral femenina, que, en promedio, posee mayores niveles de estudios que la masculina; definir y ejecutar políticas integrales que liberen a las mujeres de su responsabilidad casi exclusiva a la reproducción de la fuerza de trabajo, a través de su labor de cuidados, y que faciliten una mayor incorporación al mercado laboral y la generación de ingresos propios; y dar cumplimiento a la disposición constitucional de reconocer las labores de cuidados como un trabajo creador de valor, cuyo aporte a la producción es tarea pendiente.

El desempleo en el país continúa afectando en mayor proporción a las mujeres y a las personas jóvenes. Al 2017, la tasa de desempleo ampliado para las mujeres fue de un $22.7 \%$ y en los hombres se estima en un $10.7 \%$, lo cual evidencia que los perjuicios para las mujeres se mantienen en todos los grupos de edad y en cualquier ámbito del territorio, teniendo las mujeres rurales mayores niveles de desocupación (30.2\%) que las urbanas (22.7\%); al igual que las mujeres con estudios secundarios (28.1\%) y las pertenecientes a los grupos más pobres (42.9\% y $29.2 \%$ en los quintiles 1 y 2 ) (Banco Central de la República Dominicana, 2017). El desglose por edad y por sexo nos dice lo siguiente: mujeres jóvenes entre 15 y 19 años tienen un $55.6 \%$ de desocupación y mujeres entre 20 y 24 años tienen un $42.6 \%$ de desocupación. En contraste, en los hombres la tasa de desocupación es de un $30.1 \%$ entre $15-19$ ańos y de un $15.9 \%$ entre 20 y 24 años (Ministerio de Economía, Planificación y Desarrollo, 2018).

Las desigualdades de acceso a empleo remunerado evidencia tasas disímiles de ocupación entre hombres y mujeres. Para el 2017, la tasa de ocupación femenina fue de apenas un $45.5 \%$; en cambio, en los hombres esta inserción fue de un $73.3 \%$. La población ocupada se inserta esencialmente en el sector informal de la economía $(57.8 \%)$, con una mayor proporción de hombres (61.8\%) que de mujeres $(53.9 \%)$, sector caracterizado por bajos salarios, condiciones precarias e inestabilidad laboral (Banco Central de la República Dominicana, 2017).

Se verifica una segregación laboral por género. Las mujeres se concentran en sectores tradicionales como servicios (88.2\%), en actividades similares a las que realizan en el ámbito doméstico y comunitario, acorde al rol socialmente asignado como cuidadoras, en labores de alta desprotección (trabajo doméstico remunerado y otros tipos de servicios). También se insertan en comercio (21\%), hoteles, bares y restaurantes $(9.6 \%)$ y manufactura $(8.1 \%)$ $y$, en menor proporción, en agricultura (2\%). Mientras la inserción laboral masculina es más diversificada, esencialmente en servicios (57\%), comercio $(21.3 \%)$, agricultura $(20 \%)$, industria $(10.9 \%)$, transporte y comunicaciones $(10.8 \%)$ (Banco Central de la República Dominicana, 2017).

\section{Condiciones laborales: brechas en los ingresos laborales y en la cobertura de la seguridad social}

En las últimas décadas se verifica en el país un estancamiento de los ingresos laborales reales de la fuerza laboral, manteniéndose rezagados con relación al crecimiento de la productividad laboral. Para el período 1997-2016, el crecimiento promedio de la productividad por trabajador fue de un $2.5 \%$, mientras que el ingreso real por hora exhibe un crecimiento promedio negativo de un $0.1 \%$; es decir, en términos acumulados, el ingreso real por hora agregado experimentó una tasa de crecimiento negativa de un $8 \%$; en contraste, la productividad promedio por trabajador creció un $61 \%$. Destaca que en el período 
2011-2016 se produjo un crecimiento del salario real de un $1.2 \%$. A esta brecha entre los salarios reales y la productividad laboral se asocian factores como el poder de negociación de los trabajadores, la presión salarial a la baja que recibe la República Dominicana de países exportadores con menores salarios y el nivel de educación de la población ocupada (Lopez Hawa $\&$ Jiménez Polanco, 2017).

En noviembre de 2017, los salarios mínimos más altos pagados en República Dominicana eran de $\mathrm{RD} \$ 15,447.60$ para empresas grandes y hasta $\mathrm{RD} \$ 9,411.60$ pesos para las más pequeñas, los cuales resultan ser menores al costo de la canasta básica de bienes y servicios, estimada en $\mathrm{RD} \$ 29,013$ promedio a nivel nacional. Los hogares de menores ingresos necesitaban $\operatorname{RD} \$ 13,228$ para adquirir una cesta de alimentos aceptables para su alimentación. Tales salarios apenas permiten cubrir el $53 \%$ del costo de la canasta promedio nacional, en el caso de las grandes empresas, y el $32 \%$ en las pequeñas empresas.

A la depreciación sostenida en el poder adquisitivo de ingresos laborales de trabajadores y trabajadoras y a los bajos salarios predominantes, se adicionan diferencias salariales según género, en menoscabo de las mujeres, independientemente de la edad, el nivel educativo, el grupo y la categoría ocupacional, la zona urbana o rural, los niveles de pobreza y el sector formal e informal. Se estima que el ingreso laboral promedio mensual de la PEA femenina ocupada equivale al $83.8 \%$ del recibido por los hombres; el $90.1 \%$ en el sector formal y el $65.2 \%$ en el informal, sector que absorbe más del $53 \%$ de la mano de obra femenina ocupada. Las mayores brechas se registran en las mujeres mayores de 40 años, las que habitan en zona urbana, las que laboran en el sector servicios - especialmente en servicio doméstico, hoteles, bares y restaurantes e intermediación financiera y seguros-, en zonas francas y en el sector privado ${ }^{8}$.

8. Datos procesados por el SISDOM del Ministerio de Econo-
En términos de afiliación a un sistema de salud y de pensiones, la proporción de trabajadoras beneficiarias de este servicio supera a la de los trabajadores. Al 2015, se verifica una brecha en detrimento de las mujeres en el porcentaje de la PEA afiliada a un seguro de salud, al estimarse en un $73.3 \%$ para las mujeres y un $80 \%$ para los hombres. La menor cobertura se verifica en las mujeres menores de 19 años (36.9\%) y en las mayores de 60 años, así como en aquellas trabajadoras que habitan en la zona rural. En el caso del seguro de pensiones, este alcanza al $76.3 \%$ de las mujeres y al $70.6 \%$ de los hombres ocupados (Ministerio de Economía, Planificación y Desarrollo, 2018).

Cuando se analizan los datos provistos por el Consejo Nacional de Seguridad Social, en noviembre del año 2017, la población afiliada al sistema familiar de salud (cotizantes y dependientes) era de 7.48 millones de personas, equivalente al $73 \%$ de la población total, el $51.7 \%$ del régimen contributivo y el $47.3 \%$ del sistema subsidiado. Gran parte de las mujeres beneficiarias del SFS están afiliadas a través del régimen contributivo (51\%), fundamentalmente en calidad de dependientes (62\%), debido a la amplia disparidad entre la tasa de ocupación femenina y la masculina. De igual manera, las mujeres son mayoría en la cantidad de afiliadas en el régimen subsidiado (Ministerio de Economía, Planificación y Desarrollo, 2018).

En el Seguro de vejez y pensiones es menor el número de mujeres beneficiarias con respecto al número de hombres. Las estadísticas oficiales de la Superintendencia de Pensiones (SIPEN) evidencian que a finales de 2017 había 1.8 millones de personas cotizantes, de los cuales el $55.6 \%$ son hombres y el $44.4 \%$ restante son mujeres. Esta cobertura de seguro de vejez equivale al $36.50 \%$ de la

mía, Planificación y Desarrollo (MEPYD) al 2015, basados en la Encuesta nacional de fuerza de trabajo. Recuperado de http:// economia.gob.do/mepyd/despacho/unidad-asesora-de-analisis-economico-y-social/sisdom/sisdom-2015/ 
PEA masculina y al $42.43 \%$ de la PEA femenina ocupada (SIPEN, 2018).

También, en la afiliación al Seguro de Riesgos Laborales (SRL) se evidencia brechas de género. En este seguro, la cantidad de personas aseguradas alcanzó 2.08 millones de personas en 2017, de cuyo total el $56.6 \%$ son hombres y el $44.4 \%$ son mujeres (SIPEN, 2018).

\section{Las mujeres en las MIPYMES y en la producción agropecuaria}

La encuesta FONDOMICRO, realizada en 2013, destaca la segregación existente y la vulnerabilidad de las empresas según el género. La mayor proporción de micro y pequeñas empresas de mujeres se dedican a la elaboración de alimentos y bebidas, confección de textiles, comercio al por menor, actividades de embellecimiento y a los servicios educativos. En cambio, los hombres están más vinculados a los talleres de elaboración de productos de madera, de metalmecánica, colmados, bancas de apuestas, barberías, reparación automotriz y venta de automóviles, accesorios y repuestos. También, este estudio resalta las deficiencias en términos de manejo administrativo y la importante tasa de fracaso de las microempresas propiedad de mujeres, generalmente asociadas a desigualdades de género en lo relativo a sus responsabilidades en el trabajo de los cuidados, los estereotipos de género y las creencias y prácticas sociales predominantes (Ortiz et al., 2014).

Todavía existe un alto grado de desigualdad en la propiedad de la tierra, como uno de los principales medios productivos en las zonas rurales, lo que perjudica el estado actual de las mujeres. De acuerdo con el Pre-censo Nacional Agropecuario de 2015 , un $83.7 \%$ de las personas productoras a nivel nacional son hombres, mientras que el $16.3 \%$ son mujeres (Oficina Nacional de Estadística, 2016).

\section{Avances y desafíos en materia educativa hacia la igualdad entre hombres y mujeres}

En materia educativa, las mujeres son mayoría en casi todos los niveles educativos, incluida la educación universitaria, aunque se mantiene una segregación en la formación técnico profesional. Los principales indicadores muestran (Ministerio de Economía, Planificación y Desarrollo, 2018).

- El número medio de años de escolaridad de la población de 25 a 39 años es de 11.26 ańos para la población femenina, frente a 10.02 ańos registrado por la población masculina.

- Las mujeres tienen menor nivel de analfabetismo $(6.96 \%$ frente a $7.06 \%)$.

- La tasa neta de cobertura de educación de nivel básico ajustada arrojada es de un $98 \%$ para las mujeres, frente a un $96 \%$ para los hombres.

- Una amplia deuda pendiente es aumentar la cobertura de la educación inicial, que apenas alcanza al $32.8 \%$ de las nińas y al $34.86 \%$ de los niños.

En el ámbito de la educación universitaria, la población matriculada en 2015 fue de 480,103 personas, representando una tasa bruta de matrícula de un $37.40 \%$ para el rango poblacional entre 18 y 24 años, donde las mujeres representan el $64.4 \%$ de la matrícula universitaria. Según datos de la ENHOGAR 2015, las mujeres son mayoría en carreras como educación, psicología, enfermería y bioanálisis; en cambio, los hombres son mayoría en las carreras de ingeniería y aquellas asociadas a la tecnología (Ministerio de Educación Superior, Ciencia y Tecnología, 2016). 
Autonomía física: el derecho a la salud, el ejercicio de los derechos sexuales y reproductivos y la violencia contra la mujer

\section{Brechas en el ejercicio de los derechos sexuales y reproductivos}

La mortalidad materna es un indicador de gran relevancia para valorar el impacto de las políticas públicas en materia de salud sexual y salud reproductiva. De acuerdo con los datos del Ministerio de Salud, la República Dominicana logró descender la mortalidad materna de 101.8 en 2014 a 99.3 en 2015; y 90.1 por cada 100 mil nacidos vivos en 2016, lo que representa una reducción de un $9.2 \%$. A pesar de ello, seguimos siendo uno de los países de la región con tasas más altas, al sobrepasar el promedio de 82 por cada 100 mil nacidos vivos (Ministerio de Educación Superior, Ciencia y Tecnología, 2016).

Los niveles vergonzosos de mortalidad materna en el país están fuertemente asociados con factores como la atención médica inadecuada $(97 \%$ de los partos son institucionales y más del $80 \%$ de esas muertes son evitables), el bajo nivel educativo de la población, los altos niveles de pobreza, entre otros.

La principal causa de la mortalidad materna está relacionada con el trastorno hipertensivo en el embarazo, parto y puerperio, seguido del shock hemorrágico $(11 \%)$, la sepsis $(10 \%)$, el aborto $(7 \%)$ y las complicaciones del puerperio (7\%); mientras otras causas obstétricas indirectas aportan un 26\% al indicador. Se reconoce el aborto como una de las causas de muerte materna en el país, tal como indica la Sociedad Dominicana de Obstetricia y Ginecología al revelar que 20 de cada 100 muertes maternas son producto de abortos inseguros.

Dentro del área del ejercicio a la maternidad destaca la alta proporción de partos por cesárea $(58.1 \%$ de los partos durante 2 ańos anteriores a la encuesta) (Oficina Nacional de Estadística, 2015), que coloca a República Dominicana como el país con más alta tasa de partos por cesárea en el mundo, lo que podría prefigurar inducción por parte del personal de salud y no necesidad quirúrgica de las mujeres gestantes.

El uso de métodos anticonceptivos es un indicador importante para medir la autonomía física de las mujeres. En la ENHOGAR MICS, 2014, se reporta que solo 69 de cada 100 mujeres en edad fértil, casadas o unidas, usan algún método anticonceptivo (moderno o tradicional). República Dominicana se encuentra en un nivel intermedio de necesidad insatisfecha de anticonceptivos, comparada con otros países de América Latina. Del total de mujeres en edad reproductiva, el $68 \%$ usa métodos anticonceptivos modernos y la necesidad insatisfecha para el 2014 se estima en un $11.4 \%$ (Oficina Nacional de Estadística, 2015).

A nivel de las necesidades satisfechas de métodos modernos de planificación de la familia entre los adolescentes de 15 a 19 años, se estima en un 54,5 $\%$ y en un $60,6 \%$ entre los jóvenes de 20 a 24 años. En tal sentido, se estima que alrededor del $47.7 \%$ de todos los embarazos no son planificados, especialmente entre adolescentes y mujeres jóvenes (Oficina Nacional de Estadística, 2015).

Se subraya el gran déficit en materia de educación integral en sexualidad en todos los niveles educativos y destaca la información de UNFPA 2012 de que solo 9 de cada 100 escuelas implementó en su momento el programa de educación afectivo sexual, en el cual solo participaron 7 de cada 100 estudiantes a nivel nacional (Oficina Nacional de Estadística, 2015).

\section{VIH y SIDA}

En República Dominicana, la tasa de prevalencia general de VIH se ha reducido en la última déca$\mathrm{da}$, al estimarse un promedio de $1 \%$, siendo superior en los hombres $(0.9 \%)$ que en las mujeres $(0.7 \%)$, en 2014 . De acuerdo con las estimaciones 
y proyecciones nacionales de prevalencia de VIH y carga de enfermedad en 2014, de un total de 69,290 personas viviendo con VIH, 31,420 son mujeres y 34,710 son hombres?

En tal sentido, el país experimenta una epidemia concentrada en grupos clave, como es la población de 15 a 49 años, con el $0.8 \%$ (0.5-0.9\% en mujeres y $0.7-1.2 \%$ en hombres). La prevalencia es particularmente alta en hombres que tienen sexo con hombres $(5.2 \%)$, trabajadoras sexuales (4.5\%), migrantes haitianos (3.8\%), así como en mujeres en vulnerabilidad social (2.4\%). En el caso de las mujeres, la incidencia está vinculada a causas de carácter cultural, social y económico, las cuales se conjugan para limitar las posibilidades de que se protejan y puedan decidir por sí mismas el uso de métodos de protección, además de que también impactan en los niveles de violencia (CESDEM, 2014).

\section{Embarazo en adolescentes}

Los datos oficiales indican que el $22.3 \%$ de los nacimientos en el período 2010-2015 fueron de madres entre 15 y 19 años, estando un 34\% superior al promedio de la región de América Latina y el Caribe. La mayor incidencia se verifica en la zona sur y el Cibao central del país; más en la zona rural que en la urbana, dentro de los estratos económicos más pobres y en las adolescentes con menores niveles educativos (Oficina Nacional de Estadísticas, 2017a). Esta realidad tiene un impacto importante en sus logros educativos, en su formación e inserción laboral, en la salud sexual y reproductiva, en nuevos embarazos, en sus planes y expectativas de vidas, entre otros, tanto para ella como para el padre, cuando él también es adolescente.

El embarazo a edad temprana tiene un alto vínculo con uniones prematuras. Aunque existen

9. Datos Publicados en la página Web de la Oficina Nacional de Estadísticas. Recuperado de https:/www.one.gob.do/sociales/salud en febrero de 2018 embarazos deseados y otros no planificados, esta realidad revela severas limitaciones para el ejercicio de los derechos reproductivos entre las adolescentes. Además, se traduce en un retroceso de las ganancias, ventajas y oportunidades que genera el control sobre la reproducción y el descenso de la fecundidad a escala social, familiar e individual (Oficina Nacional de Estadística, 2017). Aunque todavía gran parte de las madres adolescentes dice haber deseado sus embarazos, no necesariamente es una expresión de una decisión planificada, sino un reflejo de las normas sociales y los roles de género.

\subsection{Violencia contra las mujeres}

Según muestran las ENDESAS -2002, 2007 y 2013-, se observa un aumento creciente en la incidencia de la violencia contra las mujeres en los últimos 15 años, especialmente la ejercida por la pareja y la expareja. La proporción de mujeres que reportaron violencia física, emocional y sexual creció de un $28 \%$ en 2002 a un $35 \%$ en 2013; mientras solo la violencia emocional se incrementó del $18 \%$ al $31 \%$, en igual período (CESDEM y ICF International, 2014). La violencia emocional es la más frecuente de todas (31\%), seguida por la violencia física (19\%) y la sexual (10\%). La incidencia de la violencia es mayor en los siguientes tipos de mujeres: las separadas, viudas o divorciadas (48\%), las que tienen cinco o más hijos/as (50\%), las que tienen educación primaria entre 5-8 años (42\%), las del quintil más pobre (40\%), las adolescentes y jóvenes (40\%) y aquellas que residen en la zona urbana (36\%) (CESDEM) y ICF International, 2014). Esta problemática en el país es el resultado del gran desequilibrio sociocultural de poder que hay entre hombres y mujeres, y del machismo preponderante como lógica social; aunque se verifica un mayor empoderamiento de las mujeres, no se percibe una mejora en las relaciones de poder, lo que repercute en más agresividad en los hombres (Pola Zapico, 2017).

Las cifras de feminicidios, como dimensión más visibilizada de la violencia, se mantienen altas, 
calculándose 177 feminicidios en promedio por año, en el período 2005-2017. En noviembre de 2017 hubo 170 feminicidios u homicidios de mujeres, de los cuales se destacan 83 feminicidios íntimos. Estos niveles colocan a la República Dominicana en la quinta posición, a nivel de Latinoamérica y el Caribe en 2016, superada solo por Honduras (466), El Salvador (371), Argentina (254) y Guatemala (211).

La cantidad de mujeres que realizan denuncias ante la justicia ha ido en aumento con los años, tanto por delito sexual como por violencia intrafamiliar y de género. La Procuraduría General de la República reportó en 2016 unas 70,864 denuncias por violencia de género e intrafamiliar; 64,423 fueron por violencia de género -física, sicológica y verbal- y 6,741 fueron denuncias por delitos sexuales. Llama la atención la baja cantidad de órdenes de protección emitidas $(15,093)$ con relación a la magnitud de las denuncias, especialmente por violencia intrafamiliar. En octubre de 2017 se había recibido más de 6,000 denuncias por violencia de género; más de 37,700 por violencia intrafamiliar y 4,350 por delitos sexuales (Procuraduría General de la República, 2018).

A pesar de estas cifras, se presume importantes subregistros, debido a la confluencia de varios factores como son la falta de confianza en la respuesta del sistema de atención (solo el 2\% de los casos es judicializado) y la escasa conciencia de lo que significa la violencia para las mujeres, considerando que la cultura predominante "naturaliza" estas actuaciones o simplemente oculta la situación.

En el sexto informe periódico de la República Dominicana sobre derechos humanos, emitido por la Comisión de Derechos Humanos de Naciones Unidas de 2017, se expresa preocupación por las elevadas tasas de violencia contra la mujer, incluyendo la violencia doméstica y el elevado número de feminicidios y violaciones anuales. También llama la atención el reducido número de sentencias y la no recepción de información estadística sobre las condenas impuestas por este tipo de violencia, creando impunidad para los autores y obstáculos para la asistencia, protección y reparación de las víctimas, así como las limitaciones de protección disponibles para ellas, incluyendo el limitado número de casas de acogida, en particular en las áreas rurales, donde son inexistentes (Consejo de Derechos Humanos de las Naciones Unidad, 2017).

Otro elemento importante por destacar son las dificultades para la aplicación de la ley, dada la visión patriarcal, debido "al enfoque androcentrista y familista que presiona a la mujer a mantenerse en una relación violenta". La baja escucha a las mujeres, donde el imputado sigue siendo el protagonista, apoyado por la interpretación de la normativa en la que muchas veces no se toma en cuenta los mandatos de las convenciones internacionales $\mathrm{y}$, si se hace, es a favor del imputado agresor. A 20 años de promulgada la Ley 24-97 sobre violencia en contra de las mujeres, se evidencia debilidades en su aplicación, por lo que se reclama la definición e implementación de un sistema integral de protección y atención a la violencia contra las mujeres como problema de interés público (Pola Zanpico, 2017).

La prevención y atención a la violencia de género y contra las mujeres y niñas requiere de cambios profundos a nivel legislativo, que implican modificar la Ley 24-97 sobre violencia contra las mujeres. Desde el año 2012, existe una propuesta de ley sometida al Congreso Nacional, elaborada con la participación de las mujeres. Esta propuesta fue aprobada recientemente en el Senado y se encuentra en la Cámara de Diputados para sanción. La propuesta aprobada incluye modificaciones a la propuesta original, evidenciando numerosos retrocesos a la inicialmente elaborada y a la ley vigente, entre los que destacan: limitar el feminicidio al ámbito íntimo y al conexo; poca vinculación a la asignación presupuestaria para atender esta problemática; débil respuesta en el ámbito local; la sanción a la violencia física y a 
la violencia psicológica se limita a la relación de pareja actual, pasada o futura; hacer equivalente la violencia sexual a la violación sexual; débiles mecanismos de participación de la sociedad civil y para rendición de cuentas, entre otras limitantes para el accionar de actores claves como la Policía Nacional y la justicia; reducir la categoría de ley orgánica a ley ordinaria, aumentando su vulnerabilidad a modificación de contenidos, entre otros.

\section{Uniones tempranas y matrimonio infantil}

La práctica nociva de matrimonio infantil sigue impidiendo que las mujeres y las niñas tengan igualdad de oportunidades, contribuye a que aumente la violencia contra las mujeres y se perpetúe el ciclo de la pobreza. Se estima en un $11.7 \%$ la proporción de mujeres dominicanas entre 18 y 22 años que se casó o que vive en pareja desde antes de cumplir 15 años, y el $35.9 \%$ de las mujeres entre 20 y 24 años declara haberse casado o estado unidas de manera estable antes de los 18 años, lo que coloca al país en la cabeza de las cifras de matrimonio infantil en América Latina. Conforme con la ENHOGAR 2014, el 35\% de las adolescentes, de 15 a 19 años, actualmente casadas o unidas, tiene parejas entre 5 y 9 años mayores que ellas y el $23.4 \%$ lo está con hombres 10 años mayores o más, lo que muestra una situación de abuso sexual, acorde con las normas establecidas (Oficina Nacional de Estadísticas, 2015).

El matrimonio infantil y adolescente es una realidad compleja que se origina por causas diversas: exclusión económica, el predominio de familias autoritarias y violentas, y la construcción de la feminidad de manera tradicional, donde la percepción de "ser mujer" en las niñas y adolescentes está asociada con ser madres-esposas; los imaginarios de la sexualidad; percepciones sobre el amor, las relaciones de pareja y el matrimonio, etc.; todo esto encuentra amplia aprobación social como resultado de prácticas adultocéntricas del patriarcado, el racismo y el heterosexismo instalados en el imaginario social (Plan Internacional República Dominicana, 2017).

\section{Inseguridad alimentaria y nutricional}

Todavía existe en el país más de un millón de personas subalimentadas, en cuyo grupo unos 65 mil niños sufren de desnutrición crónica, por una precaria ingesta de micronutrientes (Isa Contreras, 2018). Todo esto, a pesar de los progresos en materia de alimentación y nutrición ocurridos entre 1990 y 2016, donde la proporción de población subalimentada cayó de más del $32.5 \%$ a un $12.3 \%$, y la desnutrición infantil crónica (proporción de niños y niñas menores de 5 años que muestran una insuficiencia de la talla para la edad) se redujo desde más de un $20 \%$ a un 5\%, en 2013.

La prevalencia de la desnutrición infantil crónica es particularmente elevada en los nińos y las nińas de los hogares más pobres y en aquellos de madres sin educación y con educación hasta el nivel primario. Además, persisten déficits de micronutrientes importantes entre mujeres no embarazadas y en niños y niñas menores de 5 años. En adición a tales déficits, se registra un intenso crecimiento del sobrepeso y la obesidad, estimándose que cerca del 39\% de los hombres y más del $50 \%$ de las mujeres entre 15 y 49 años estaba en sobrepeso u obesidad.

Este tipo de problemática requiere de diversas intervenciones que van desde el fortalecimiento y la puesta en funcionamiento de un marco legal acorde a esta nueva realidad; la mejora de la calidad de los programas, diseñándolos mejor y focalizando su atención en los temas que reciben menos atención y garantizando el enfoque de género; la generación de evidencias para la toma de decisiones; asegurar los recursos para su implementación sostenible; la construcción de mejores instituciones; fortalecer la coordinación interinstitucional dentro del sector público y privado, y el desarrollo de acciones formativas.

La transversalización del enfoque de género en esta ruta recién definida para trabajar este tema es una condición indispensable para garantizar la 
reducción de las inequidades de género, generacional y de los grupos más desfavorecidos.

\section{Baja participación femenina en las estructuras de poder}

Se continúa verificando una baja participación en la representación de las mujeres en los espacios políticos de toma de decisiones, a pesar del aumento de las mujeres en las candidaturas y de tener un mayor nivel educativo con relación a los hombres. Por ejemplo, la junta monetaria, donde se toman las principales decisiones de índole económica, está compuesta por 10 integrantes, dentro de los cuales solo hay una mujer y esta funge como secretaria. En el poder ejecutivo, si bien una mujer ocupa el cargo de vicepresidenta de la República, la participación femenina continúa siendo minoría a nivel ministerial, pues apenas 4 mujeres ocupan los puestos de ministras de 22 ministerios existentes -en áreas de la educación superior, ministerio de la mujer, salud y juventud-, realidad que se ha mantenido por décadas, a pesar de que las mujeres constituyen alrededor del $63.6 \%$ del total de la nómina del sector público ${ }^{10}$.

A nivel de las personas que laboran en el Congreso Nacional, en la Cámara de Diputados las mujeres ocupaban el $28.1 \%$ de los escaños en 2016, mientras en el Senado apenas hay 3 senadoras, de 32 puestos, evidenciando que aún no se ha logrado el cumplimiento de la Ley 12-2000 sobre el 33\% de la cuota femenina. Situación similar se observa en el poder local, donde solo se ha podido lograr la cuota a nivel de regidurías (34.1\%), y en cuanto a las alcaldías, apenas el $12.1 \%$ son gerenciadas por mujeres.

Los obstáculos que enfrentan las mujeres para competir en los puestos electivos se originan en

10. Datos calculados por el Ministerio de Administración Pública, en julio de 2017. Recuperado en febrero de 2018 de https://map.gob.do/ factores como: sistema de partidos políticos que opera bajo la lógica patriarcal; contexto político que no promueve la paridad; sistema electoral que afecta negativamente a las mujeres para competir en igualdad de condiciones que los hombres; la persistencia de una cultura política y de prácticas clientelares que demandan cada vez más recursos; entre otros. Estas situaciones muestran cómo los sistemas electorales no son neutros, ni en sentido general ni en lo relativo al género, aumentando la desventaja para las mujeres (Duarte \& Hasbún, 2009). La división sexual del trabajo y los roles de cuidado socialmente asignados a las mujeres limitan sus posibilidades de participación política, tanto fuera como dentro de los partidos y se reflejan en los mecanismos que se establecen al interno de los partidos -nominaciones bloqueadas, cuotas reservadas- que impiden el avance de las mujeres en las nominaciones para puestos de poder dentro de las estructuras partidarias, en los puestos electivos y en el poder ejecutivo.

Se observan algunos pasos de avance en la participación de las mujeres en los estamentos de poder para la promoción de una agenda de equidad de género en la Cámara de Diputados, así como el aumento en la participación de la mujer en las comisiones de trabajo de ambas cámaras, pero aún las mujeres están ausentes de las presidencias de las comisiones de finanzas, presupuesto, fuerzas armadas, policía, deportes, lo que claramente refleja la división sexual del trabajo legislativo y limita la inclusión de estos temas en áreas relacionadas con la economía, el desarrollo, la tecnología, entre otras.

Se identifican importantes desafíos para lograr la paridad en la participación política de las mujeres, acciones que abarcan: la aprobación y reglamentación de la Ley de partidos con enfoque de paridad de género a nivel político, sobre todo para las candidaturas a cargos congresuales y municipales; disponer de políticas para la formación en igualdad de género y derechos humanos a nivel interno de los partidos políticos; establecer sanciones por 
incumplimiento y restringir la entrega de fondos estatales si no se cumple con la capacitación, entre otras.

\section{Conclusiones y recomendaciones}

El análisis que se muestra en este documento evidencia la persistencia de desigualdades por razones de género en la República Dominicana, las cuales continúan privando a mujeres y niñas de derechos y oportunidades fundamentales. Para eliminar estas desigualdades y aumentar el empoderamiento de las mujeres y niñas se requiere de esfuerzos más efectivos y enérgicos. Para avanzar hacia la igualdad se precisa de:

- La definición y la ejecución de políticas públicas que tomen en cuenta las necesidades y vulnerabilidades de hombres y mujeres durante todo el ciclo de vida, identificando riesgos y brechas en función del género y de la edad, priorizando intervenciones claves y mejorando el uso de recursos escasos.

- La revisión de las políticas y los programas en ejecución y la elaboración y puesta en ejecución de nuevas políticas, junto a la aprobación de nuevos marcos jurídicos, para combatir la discriminación profundamente enraizada en los planos sociales, económicos y culturales, como resultado de la división sexual tradicional de trabajo, de las actitudes patriarcales y de las normas sociales que estas conllevan.

- La formulación de políticas universales de salud, educación y erradicación de la pobreza con sistemas de protección social universal, especialmente para la nińez, la adolescencia y la adultez mayor.

- La garantía de las tres autonomías de las mujeres -física, económica y política- y el fortalecimiento de los sistemas estadísticos sensibles al género, visibilizando a las poblaciones más vulnerables.
- En las reformas legislativas se precisa superar los persistentes vacíos que afectan a las mujeres y que limitan el ejercicio de sus derechos humanos, tales como:

- La prohibición absoluta del aborto y, en consecuencia, la aprobación de la despenalización en tres causales en el Código Penal.

- El cumplimiento del mandato constitucional de la laicidad del Estado.

- La promulgación de una ley integral para la prevención, atención y sanción a la violencia de género.

- La aprobación de una ley de salud sexual y reproductiva que promueva el ejercicio de los derechos sexuales y reproductivos.

- Legislaciones referentes a la conciliación entre la esfera laboral y la familiar y, en particular, el reconocimiento de la igualdad de derechos de las trabajadoras domésticas.

- La implementación de políticas de educación integral sobre sexualidad.

- La superación de debilidades en la aplicación de las normas existentes y la reglamentación de la participación política de las mujeres en condiciones de paridad.

Existe el convencimiento de que la República Dominicana, al igual que otros países del mundo, no podrá avanzar en el desarrollo humano y sostenible si las políticas públicas no acompañan a las personas en las diferentes etapas de sus vidas, respondiendo a los riesgos y las vulnerabilidades particulares de cada una de ellas. Este momento representa una oportunidad para llamar la atención a las autoridades y a los movimientos sociales sobre la gravedad de los problemas de la desigualdad, la inequidad y la exclusión de las mujeres en el ejercicio de su ciudadanía. 


\section{Referencias bibliograficas}

Banco Central de la República Dominicana (2017). Estadísticas Económicas y Mercado de Trabajo. Recuperado de https://www.bancentral.gov.do/ estadisticas_economicas/mercado_trabajo/

Comisión Económica para América Latina (2014). CEPAL. Datos y estadisticas. Recuperado en febrero de 2018 de http://estadisticas.cepal.org/cepalstat/WEB_CEPALSTAT/estadisticasIndicadores. asp?idioma $=\mathrm{e}$

Consejo de Derechos Humanos de las Naciones Unidas (2017). Sexto informe periódico de la República Dominicana sobre derechos humanos. Recuperado de http:// www.ohchr.org/SP/HRBodies/HRC/Pages/ Home.aspx

Centro de Estudios Sociales y Demográficos (CESDEM) y ICF International. (2014). Encuesta demográfica y de salud 2013. Santo Domingo, República Dominicana: CESDEM y ICF International.

Duarte, I. \& Hasbún, J. (2009). Mujer y politica en la República Dominicana: consensos y disensos entre las líderes y la ciudadanía. Santo Domingo, República Dominicana: Editora Ortega.

Isa Contreras, P. (24 de febrero de 2018). Para erradicar el hambre en República Dominicana. El Caribe. Recuperado en febrero de 2018 de: http:// www.elcaribe.com.do/2018/02/24/para-erradicar-el-hambre-en-republica-dominicana/

López Hawa, N. \& Jiménez Polanco, M. (2017). Explicando la brecha entre el salario real y la productividad laboral en la República Dominicana: análisis macroeconómico y recomendaciones de politicas basadas en microsimulaciones. Primer lugar Concurso Anual de Economía. Santo Domingo: Biblioteca Juan Pablo Duarte del Banco Central de la República Dominicana.
Ministerio de Economía, Planificación y Desarrollo. (2018). 5to Informe anual de avance en la implementación de la Estrategia nacional de desarrollo 2030 y cumplimiento de los objetivos y metas del Plan plurianual del sector público. Santo Domingo: Ministerio de Economía, Planificación y Desarrollo (MEPYD). Recuperado de http://economia.gob.do/mepyd/despacho/unidad-asesora-de-analisis-economico-y-social/sisdom/ sisdom-2015/

Ministerio de Educación Superior, Ciencia y Tecnología. (2016). Informe general sobre estadisticas de Educación superior 2015 y Resumen histórico 2005-2015. Santo Domingo: el Autor.

Oficina Nacional de Estadística (ONE). (2015). Encuesta nacional de hogares de propósitos múltiples 2014: resultados principales. ENHOGAR - MICS 2014. Santo Domingo: Oficina Nacional de Estadísticas de la República Dominicana.

Oficina Nacional de Estadística (ONE). (2016). Precenso Nacional Agropecuario 2015. Informe de resultados. Santo Domingo: Oficina Nacional de Estadística. Recuperado de https://www.one.gob. do/Categoria/Estadisticas/

Oficina Nacional de Estadística (ONE). (2017). Encuesta nacional de hogares de propósitos múltiples (ENHOGAR-2016). Informe de resultados principales. Santo Domingo: Oficina Nacional de Estadística. Recuperado de https://www.one.gob. do/Categoria/Estadisticas/

Oficina Nacional de Estadísticas (ONE). (2017a). Tendencias, patrones y determinantes de la fecundidad adolescente en la República Dominicana. Santo Domingo: UNFPA- ONE.

Ortiz, M., Cabal, M. \& Mena R. (2014). Micro, pequeñas y medianas empresas en la República Dominicana 2013. Santo Domingo: FONDOMICRO.

Pola Zanpico, M. (2017). Logros legislativos, promesas incumplidas: brechas en la implementación 
de la ley sobre la violencia contra mujeres. Santo Domingo: Oxfam.

Plan Internacional República Dominicana. (2017). Niñas esposadas: caracterización del matrimonio infantil forzado en las provincias de Azua, Barahona, Pedernales, Elías Piña y San Juan. Boletín Planteamientos, No. 2. Santo Domingo: Plan Internacional República Dominicana.

Programa de las Naciones Unidas para el Desarrollo -PNUD-. (2016). Informe regional sobre desarrollo humano para América Latina y el Caribe. Progreso multidimensional: bienestar más allá del ingreso. New York. Recuperado de http://www.pnud.org. br/Arquivos/HDR2016.pdf

Programa de Naciones Unidas para el Desarrollo. (2016). Desarrollo humano en República
Dominicana. Santo Domingo: Programa de Naciones Unidas para el Desarrollo.

Programa de Naciones Unidas para el Desarrollo. (2017). Desarrollo humano en República Dominicana. El embarazo en adolescente: un desafio multidimensional para generar oportunidades en el ciclo de vida. Santo Domingo: Programa de Naciones Unidas para el Desarrollo.

Procuraduría general de la República (2018). Portal de Transparencia. Santo Domingo: Recuperado en febrero de 2018 de http://transparencia.pgr.gob. do/GestorArchivos/Index/4339 e

Superintendencia de Pensiones (SIPEN) (2018). Boletin de Datos Estadísticos. Santo Domingo: SIPEN. Recuperado en febrero de 2018 de www. sipen.gob.do/boletin/datos_estadisticos.php 